\title{
Method of Parameters Determination for Multi-Winding Transformer Equivalent Circuit in the Form of Multi-Beam Star
}

\author{
Suslov V.M. Bosneaga V.A. \\ Institute of Power Engineering \\ Kishinau, Republic of Moldova
}

\begin{abstract}
For the case of transformer with a number of windings four or more, the number of pair short-circuit impedances are more than the number of beams of the corresponding star, which makes it impossible to obtain a sufficiently correct equivalent scheme in the form of a multi-beam star without electromagnetic coupling between the elements of the beams. The scope of this paper is the elaboration of the technique for determining the elements of the equivalent circuit for the replacement of a multi-winding transformer in the form of multi-beam star with mutual electromagnetic coupling between the elements of the beams, with one magnetization branch. The scope of the article is achieved by application of the formula that connects the pair short-circuit impedance of the two windings with their total own and mutual impedance. Using the matrix pseudo-inversion procedure, a technique was developed for finding the own and mutual impedances, constituting the star's beams. The new results consist in the fact that this equivalent scheme is universal and did not depend on the specific mutual arrangement of the windings. Besides, it is possible to determine the parameters of the proposed multi-beam equivalent circuit in exact accordance with the values of the pair short-circuits impedances of the transformer and is a logical continuation of the conventional equivalent scheme of three-winding transformer. In addition, the proposed algorithm ensures the positive values of the own inductive resistances of the beams (windings).
\end{abstract}

Keywords: equivalent scheme, multi-beam star, pair short-circuit windings impedances.

DOI: $10.5281 /$ zenodo. 1343402

\author{
Metoda determinării parametrilor circuitului echivalent a transformatorului \\ cu mai multe înfășurări sub forma stelei multi-pate \\ Suslov V.M., Bosneaga V.A. \\ Institutul de Energetică \\ Chișinău, Republica Moldova
}

Rezumat. Este cunoscută metoda construirii schemelor echivalente pentru transformatoare cu două sau trei înfășurări sub formă de stea (fără legături electromagnetice între elementele razelor), cu conectarea ramurii de magnetizare la un punct comun. În cazul unui transformator cu un număr de înfăşurări patru sau mai multe, numărul impedanțe de scurt-circuit a perechilor de înfășurări este mai mare, decât numărul de raze ale starului corespunzător, ceea ce face imposibilă construirea unei scheme suficient de corecte sub forma unei stele multipate fără legături electromagnetice între elementele razelor. Este de asemenea utilizat un circuit echivalent sub forma unui poligon complet cu un număr de vârfuri egal cu numărul de înfășurări ale transformatoarelor sau de scheme echivalente asemănătoare copacilor care sunt aproximative în natură. Sunt de asemenea cunoscute schemele echivalente în forma de lanțului cu influență electromagnetică a elementelor. $\mathrm{Cu}$ toate acestea, amplasarea elementelor circuitului echivalent este legată de aranjamentul reciproc al înfășurărilor transformatorului în fereastra miezului magnetic. În toate aceste cazuri, apare problema alegerii locului conectării ramificaţiei de magnetizare. În plus, aceste scheme (cu excepția schemei poligonale complete) nu sunt universale, iar metodele de determinare a parametrilor acestora sunt complexe. În această lucrare se propune metoda de determinare a elementelor schemei echivalente unui transformator cu multiple înfășurări sub forma unei stele multi-pate cu legături electromagnetice între elementele razelor, cu o ramură de magnetizare. Ca baza este folosita formula, care exprima impedanța de scurtcircuit a perechii de înfășurări prin impedanțele proprie și reciprocă înfășurărilor. Folosind procedura de pseudo-inversiune a matricei, se propune o metoda pentru determinarea impedanțelor proprie și reciproce a elementelor, care constituie razele stelei. Această schemă este universală și nu este legată de aranjamentul specific al înfășurărilor pe miez, permite determinarea parametrii circuitului echivalent în forma de stea multilaterala cu legături electromagnetice intre razele în conformitate exactă cu valorile impedanțelor de scurt-circuit a perechilor de înfășurări ale transformatorului și este o continuare logică a schemei echivalente convențională a transformatorului cu trei înfășurări. În plus, algoritmul propus asigură pozitivitatea impedanțelor inductive ale razelor stelei.

Cuvinte-cheie: circuit echivalent, stea cu mai multe raze, impedanțe de scurtcircuit a perechilor de înfăşurări. 


\section{Методика определения параметров схемы замещения многообмоточного трансформатора в виде многолучевой звезды Суслов В.М., Бошняга В.А. \\ Институт Энергетики \\ Кишинев, Республика Молдова}

Аннотация. Известна методика построения схем замещения для однофазных трансформаторов, имеющих две либо три обмотки в виде двухлучевой или трехлучевой звезды (без электромагнитных связей между элементами лучей), с подключением ветви намагничивания в общую точку. Для случая трансформатора с числом обмоток четыре и более число опытов парных короткого замыкания больше числа лучей соответствующей звезды, что делает невозможным построение достаточно корректной схемы в виде многолучевой звезды без электромагнитных связей между элементами лучей. Известны цепочечные схемы замещения со взаимным электромагнитным влиянием элементов схемы. Однако расположение элементов схемы замещения привязано к взаимному расположению обмоток трансформатора в окне магнитопровода. Во всех этих случаях возникает проблема выбора места подключения ветви намагничивания. Кроме того, данные схемы (за исключением схемы полного эквивалентного многоугольника) не носят универсального характера, и методики определения их параметров сложны. Целью настоящей работы является разработка методики определения элементов схемы замещения многообмоточного трансформатора в виде многолучевой звезды со взаимными электромагнитными связями между элементами лучей, с одной ветвью намагничивания. Поставленная цель достигается путем использования формулы, связывающей парное сопротивление короткого замыкания двух обмоток с их полными собственными и взаимным сопротивлением. С использованием процедуры псевдо-обращения матрицы построена методика нахождения собственных и взаимных сопротивлений, составляющих лучи звезды. Новизна предложенной схемы заключается в том, что данная схема универсальна, и не связана с конкретным взаимным расположением обмоток, обеспечивается определение параметров предлагаемой многолучевой схемы замещения в точном соответствии со значениями парных сопротивлений короткого замыкания трансформатора и является логическим продолжением общепринятой схемы замещения трехобмоточного трансформатора. Кроме того, предложенный алгоритм обеспечивает положительность собственных индуктивных сопротивлений лучей (обмоток).

Ключевые слова: эквивалентная схема, многолучевая звезда, парные сопротивления короткого замыкания.

\section{Введение}

В большинстве литературных источниках по силовым трансформаторам (например, [13]) описывается теория трансформаторов, включая построение схем замещения, однофазных трансформаторов, имеющих две либо три обмотки. Схему замещения для таких трансформаторов представляют в виде двух или трех лучевой звезды (без электромагнитных связей между элементами лучей), с подключением ветви намагничивания в общую точку, где соединены все лучи. Возможность построения такой схемы для двух- или трехобмоточного трансформаторов очевидна.

Для случая трансформатора с числом обмоток четыре и более число опытов парных коротких замыканий (к.з.) больше числа лучей соответствующей звезды, что делает невозможным построение достаточно корректной схемы в виде звезды без электромагнитных связей между элементами лучей. Принято параметры элементов предлагаемых схем замещения $n$ обмоточного трансформатора определять из совокупности опытов парного короткого замыкания обмоток, при подаче напряжения на одну из них, обеспечивая их номинальную загрузку по току (в случае если обмотки разной мощности, обеспечивают номинальную загрузку обмотки меньшей мощности). При этом считают допустимым не учитывать влияние ветви намагничивания на определяемые параметры.

Для случая многообмоточных трансформаторов, большинство авторов [9-23] предлагают схему полного многоугольника с количеством вершин, равным числу обмоток трансформатора. Приближенный характер носят древовидные схемы замещения, описанные в [9,12]. В [14,15] приводится вариант цепочечной схемы замещения с количеством элементов цепочки на единицу меньшим числа обмоток с взаимным влиянием друг на друга. В данном варианте расположение элементов схемы замещения привязано к взаимному расположению обмоток трансформатора в окне магнитопровода. Во всех этих случаях возникает проблема выбора места подключения ветви намагничивания, и 
данные схемы (за исключением схемы полного многоугольника) не носят универсального характера. Некоторые же предлагают схему многолучевой звезды с тем же количеством лучей, но не связанных электромагнитно между собой, причем без адекватного обоснования такого допущения $[16,17]$. Вариант схемы замещения близкий по геометрическому начертанию с предлагаемым, описан в [18], однако методика определения элементов схемы замещения непрактична. Кроме всего, предлагаемые методики определения элементов всех этих схем замещения крайне усложнены.

\section{РАСЧЕТ ПАРАМЕТРОВ СХЕМЫ ЗАМЕЩЕНИЯ}

В настоящей работе предложена простая методика определения величин элементов предлагаемого варианта схемы замещения многообмоточного трансформатора в виде многолучевой звезды со взаимными электромагнитными связями между элементами лучей, которая имеет одну ветвь намагничивания. Как это обычно делается, будем основываться на данных, полученных из опытов парного короткого замыкания. Данная схема является универсальной. То есть, она не связана непосредственно с конкретным взаимным расположением обмоток. В соответствии с [11], после перехода к приведенным значениям параметров схема замещения однофазного двухобмоточного трансформатора может быть представлена в виде, изображенном на рис.1.

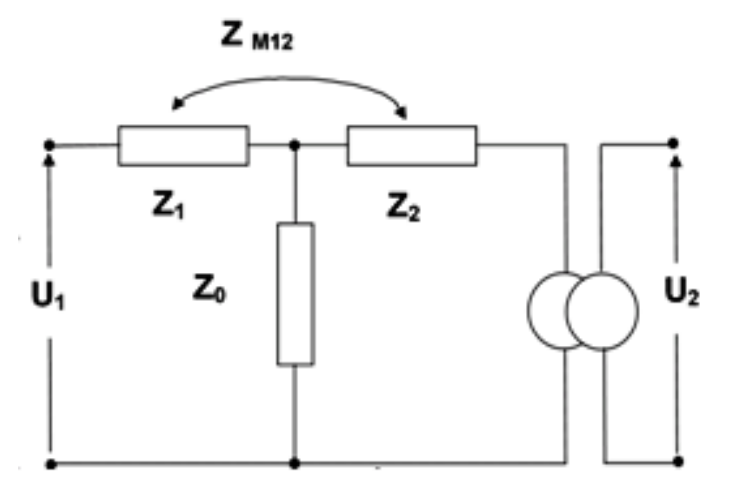

Рис.1.Схема замещения двухобмоточного трансформатора.

Здесь показаны: $U_{1}-$ комплекс напряжения источника питания, подключенного к первичной обмотке,
$U_{2}$ - комплекс напряжения, снимаемого со вторичной обмотки идеального трансформатора с тем же коэффициентом трансформации, что и замещаемый трансформатор, $Z_{1}$ и $Z_{2}$ комплексные, собственные сопротивления обмоток трансформатора, $\mathrm{Z}_{\mathrm{M} 12}$ комплексное сопротивление, отражающее взаимное размагничивающее влияние обмоток трансформатора друг на друга, $Z_{0}-$ комплексное сопротивление ветви намагничивания трансформатора. Идеальный трансформатор с тем же коэффициентом трансформации, что и замещаемый, включен на выходе схемы для получения реальных (в отличие от приведенных) значений токов и напряжений на выходе.

Согласно данной схеме замещения сопротивление, вносимое трансформатором при подключении источника питания к 1-й обмотке трансформатора при короткозамкнутой 2-й его обмотке (без учета сопротивления ветви намагничивания) определяется из следующего выражения.

$$
z_{k 12}=z_{1}+z_{2}-2 \cdot z_{M 12}
$$

Основываясь на данном соотношении, опишем методику построение схемы замещения многообмоточного трансформатора, имеющей вид, аналогичный показанному выше для двухобмоточного трансформатора. В ней уже нельзя будет (аналогично тому, как это делается в случае трансформатора с двумя или тремя обмотками) путем каких-либо преобразований избавиться от всех взаимных связей между лучами этой схемы. Данная схема показана на рис. 2.

Здесь:

n - число обмоток трансформатора;

$Z_{0}$ - комплексное сопротивление ветви намагничивания трансформатора;

$Z_{i} \quad$ - комплексное собственное сопротивление $i$-й обмотки;

$Z_{m i j}$ - комплексное сопротивление, определяющее взаимное размагничивание $i$-й и $j$-й обмоток (все эти сопротивления приведены к первичной обмотке трансформатора).

Определение параметра холостого хода трансформатора (сопротивление $Z_{0}$ ) производится традиционным способом из опыта холостого хода и здесь не рассматривается. 


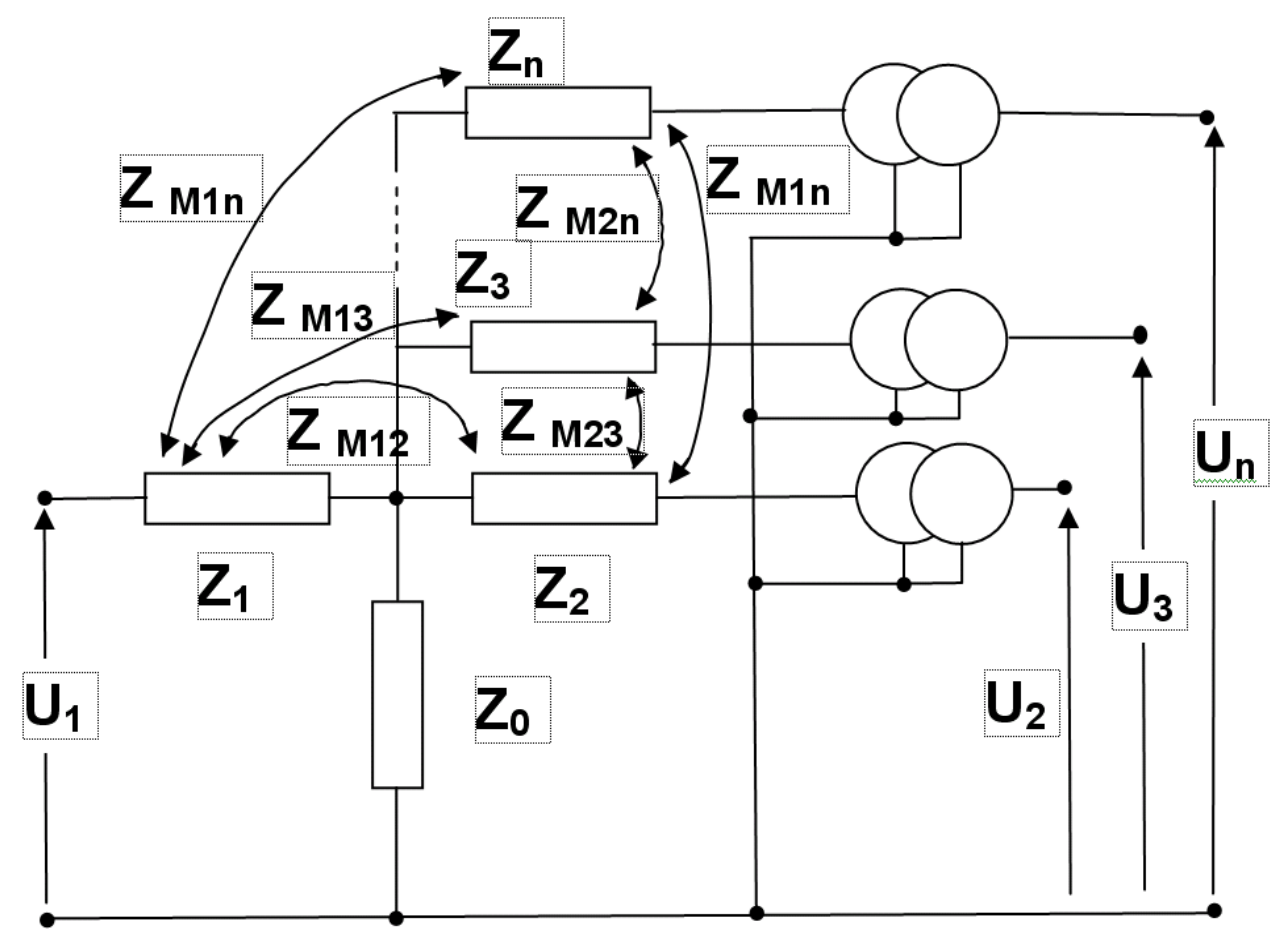

Рис.2. Схема замещения n-обмоточного трансформатора

Параметры короткого замыкания трансформатора определяются также традиционным способом на основе опытов короткого замыкания пар обмоток трансформатора.

Аналогично уравнению (1) для предыдущего случая двухобмоточного трансформатора, комплексное эквивалентное сопротивление пары ( $i$-й и $j$-й $)$ коротко замкнутых обмоток многообмоточного трансформатора определяется из следующего выражения:

$$
z_{k i j}=z_{i}+z_{j}-2 \cdot z_{M i j}
$$

Распространим это соотношение на совокупность всевозможных опытов парного короткого замыкания n-обмоточного трансформатора. Получим следующую матричную систему уравнений (3):

$$
\left\{\begin{array}{l}
\left\|C_{i j}^{\prime}\right\| \cdot\left\|z_{j}\right\|=\left\|z_{k i}\right\| \\
i=1,2 \ldots l ; j=1,2 \ldots n
\end{array}\right\}\left\{\begin{array}{l}
\left\|z_{i}\right\|^{d} \cdot\left\|C_{i j}\right\|+\left\|C_{i j}\right\| \cdot\left\|z_{i}\right\|^{d}-2 \cdot\left\|z_{M i j}\right\|=\left\|z_{k i j}\right\| \\
C_{i j}=1(i \neq j) \\
C_{i i}=0 \\
z_{k i i}=0 \\
i=1,2 \ldots n ; j=1,2 \ldots n
\end{array}\right\}
$$

Здесь: $n$-число обмоток,

$i, j$ - номера обмоток, участвующих в $\mathrm{n} *(\mathrm{n}-1)$ опытах парного короткого замыкания,

$\left\|z_{i}\right\|^{\mathrm{d}}$ - диагональная матрица комплексных собственных сопротивлений обмоток,

$\left\|z_{\text {мij }}\right\| \quad$ - матрица комплексных сопротивлений, характеризующих взаимное размагничивание $i$ - $\breve{u} u j$-й обмоток,

$\left\|z_{k i j}\right\|$ - матрица комплексных эквивалентных сопротивлений трансформатора во всевозможных парных опытах короткого замыкания,

$\left\|C_{i j}\right\|$ - матрица, характеризующая участие обмоток в опытах их парного короткого замыкания, состоящая из единиц, за исключением элементов главной диагонали (все три последние матрицы - квадратные с нулями на главной диагонали),

Полученная система уравнений (3) относительно неизвестных матриц $\left\|Z_{i}\right\|$ и $\left\|Z_{m i j}\right\|$ является недоопределенной (число 
неизвестных, подлежащих определению больше числа уравнений и равно $n^{*}(n-1) / 2$ ) и поэтому имеет бесконечное множество решений. Однако из всего множества возможных еe решений, выберем представляющее наибольший интерес то решение, которое обеспечивает минимальную степень взаимного влияния обмоток друг на друга. Это может быть обеспечено в два этапа. Сначала пренебрежем сопротивлениями взаимного влияния обмоток друг на друга. Затем, используя принцип взаимности, развернем эту систему. Получаем следующую систему уравнений.

$$
\left\{\begin{array}{l}
\left\|C_{i j}^{\prime}\right\| \cdot\left\|z_{j}\right\|=\left\|z_{k i}\right\| \\
i=1,2 \ldots l ; j=1,2 \ldots n
\end{array}\right\}
$$

где $l$ - количество опытов парного короткого замыкания, здесь уже равное $0,5 \cdot n \cdot(n-1)$, то есть в 2 раза меньшее, чем в предыдущем случае,

$n$ - число обмоток,

$i$ - номер опыта парного короткого замыкания,

$j$ - номер обмотки,

$\left\|z_{j}\right\|$ - столбцовая матрица комплексных собственных сопротивлений обмоток,

$\left\|C^{\prime}{ }_{i j}\right\|$ - новая прямоугольная матрица (отличающаяся от предыдущей матрицы участия другим расположением элементов) участия обмоток в парных опытах короткого замыкания $\left(C_{i j}=0\right.$, если $j$-я обмотка не участвует в текущем $i-$ м опыте или $C_{i j}=1-$ в противном случае),

$\left\|z_{k i}\right\|$ - столбцовая матрица комплексных эквивалентных сопротивлений трансформатора во всех парных коротких замыканиях.

Данная система уравнений теперь уже становится переопределенной (количество опытов к.з. больше числа обмоток) и, следовательно, несовместной. Тем не менее, она, согласно с [24], может быть разрешена относительно матрицы $\left\|z_{j}\right\|$ с максимальной степенью соответствия имеющимся данным парных опытов короткого замыкания. Данная процедура может быть записана в следующем виде:

$$
\left\|z_{j}\right\|=\left\|C_{i j}\right\|^{+}\left\|z_{k i}\right\|
$$

Здесь + - означает процедуру псевдообращения матрицы.

Таким образом, в результате решения системы уравнений (4) могут быть получены значения комплексных собственных сопротивлений обмоток.

Окончательно, с учетом найденных величин матрицы $\left\|z_{j}\right\|$ из (5) для каждого элемента взаимной связи обмоток согласно (2) получим:

$$
z_{M i j}=0,5 \cdot\left(z_{i}+z_{j}-z_{k i j}\right)
$$

Таким образом, по предложенной методике могут быть определены параметры всех элементов предложенной многолучевой схемы замещения трансформатора, в том числе элементов взаимных размагничивающих связей между ними (за исключением параметра ветви намагничивания).

Bce эти лучи исходят из одной точки (напряжение в которой можно трактовать как величину э.д.с. намагничивания), к которой подключается ветвь намагничивания. К концам лучей всех обмоток (кроме первичной) подключены идеальные трансформаторы с требуемыми коэффициентами трансформации.

Необходимо отметить, что данная методика обеспечивает определение параметров предлагаемой схемы замещения в точном соответствии со значениями сопротивлений во всех опытах парного короткого замыкания и является логическим продолжением общепринятой схемы замещения трехобмоточного трансформатора.

Данный алгоритм обеспечивает положительность собственных параметров лучей (обмоток) и, как правило, их превалирование над взаимными параметрами, которые могут иметь разные знаки.

Ниже приведены в таблицах 1 и 2 в качестве примера исходные данные (второй столбец таблицы 1 соответствует заданным парным сопротивлениям обмоток) и результаты расчета параметров для многолучевой схемы замещения 5-ти обмоточного трансформатора.

Значения суммарных эквивалентных сопротивлений трансформатора в опытах парного короткого замыкания взяты из [15]. 
Таблица 1.

Парные сопротивления короткого замыкания обмоток и взаимная индуктивность обмоток.

\begin{tabular}{|l|c|c|}
\hline $\begin{array}{l}\text { В опыте } \\
\text { парного } \\
\text { к.3. } \\
\text { участвуют } \\
\text { обмотки }\end{array}$ & $\begin{array}{l}\text { Суммарная } \\
\text { эквивалентная } \\
\text { индуктивность } \\
\text { обмоток, мкГн }\end{array}$ & $\begin{array}{l}\text { Взаимная } \\
\text { индуктивн. } \\
\text { между } \\
\text { обмотками, } \\
\text { мкГн }\end{array}$ \\
\hline $1-2$ & 1.1 & 0.629 \\
\hline $1-3$ & 2.24 & -0.055 \\
\hline $1-4$ & 2.95 & -0.295 \\
\hline $1-5$ & 3.55 & -0.28 \\
\hline $2-3$ & 0.864 & -0.042 \\
\hline $2-4$ & 1.595 & -0.292 \\
\hline $2-5$ & 2.23 & -0.295 \\
\hline $3-4$ & 0.675 & 0.055 \\
\hline $3-5$ & 1.33 & 0.042 \\
\hline $4-5$ & 0.58 & 0.532 \\
\hline
\end{tabular}

Таблица 2. Собственные индуктивности обмоток.

\begin{tabular}{|c|c|}
\hline № обмотки & $\begin{array}{l}\text { Собственная } \\
\text { индуктивность } \\
\text { обмотки, мкГН }\end{array}$ \\
\hline 1 & 1.854 \\
\hline 2 & 0.504 \\
\hline 3 & 0.277 \\
\hline 4 & 0.507 \\
\hline 5 & 1.137 \\
\hline
\end{tabular}

\section{ЛИТЕРАTУРА (REFERENCES)}

[1] Neiman L.R., Demirchean K.S. Teoreticheskie osnovi electrotekhniki. Chasti 2, Teoria lineinikh elektricheskikh tsepei. [Theory of electrical circuits, part 2, Theory of linear circuits]. Energia, М-Л, 1966. 227 pp. (In Russian).

[2] Elektrotekhnicheskii

spravochnik

[Electrotechnical reference book], 4-e izdanie, editor Grudinskii P.G., volume 1, M, Energia, 340 pp. (In Russian).

[3] Voldek A.I. O skheme zameschenia transformatora i ee parametrakh. Elektrichestvo, 1952, No.8, pp. 21-25. (In Russian).

[4] A.G. Ganz, "A simple, exact equivalent circuit for the three-winding transformer," IRE Trans. Component parts, vol. 9, issue 4, pp 212-213, December, 1962.

[5] Leon F., Martinez J.A. Dual Three-winding Transformer Equivalent Circuit matching Leakage measurements. IEEE transactions on power delivery. January 2009. Vol. 24. No. 1. P. 160-168.

[6] Juan A. Martinez-Velasco, Bruce A. Mork. Transformer Modelling for Simulation of Low
Frequency Transients in Power Systems. CIRED. 17th International Conference on Electricity Distribution Barcelona, 12-15 May 2003. Session 1 Paper No 92.

[7] Lambert Mathieu. Transformer Modeling For Low- And Mid-Frequency Electromagnetic Transients Simulation, PhD thesis, Universite De Montreal. 2014.

[8] J. A. Martinez and B. A. Mork, "Transformer modeling for low- and mid-frequency transients-a review," IEEE Transactions of Power Delivery, vol. 20, no. 2, pt. 2, pp. 1625-1632, Apr. 2005.

[9] Magnetic Circuit and Transformers. The Massachusetts Institute of Technology. 1943.

[10] D. Maksimovic, R. Erickson. A MultipleWinding Magnetics Model Having Directly Measurable Parameters. Power Electronics Specialists Conference, 1998.

[11] Voldek A.I. Electricheskie mashini [Electrical machines], "Energia", 1978. (In Russian).

[12] Leites L.V., Pintsov A. M. Skhemi zameschenia mnogoobmotochnikh transformatorov [Equivalent schemes of multiwinding transformers], "Energia", 1974. (In Russian).

[13] Vasiutinskii S.B. Osnovi teorii i rascheta transformatorov [Bases of theory and design of transformers], "Energia”, Л. 1970. (In Russian).

[14] Zakoriukin V.P. Algoritm rascheta skhemi zameschenia trekhfaznogo transformatora $\mathrm{v}$ faznikh koordinatakh [Algorithm of calculation of equivalent scheme of three phase transformer in phase coordinates]// Novie tehnologii upravlenia I metodi analiza electricheskih sistem I sistem teagovogo elektrosnabjenia. Irkutsk 2000. Issue 1, pp. 31-38. (In Russian).

[15] C. Alvares-Marino, F. de Leon, X.M.LopezFernandes. Equivalent Circuit for the Leakage Inductance of Multiwinding Transformers: Unification of Terminal and Duality Models. IEE Transaction on Power Delivery, vol. 27, NO 1, January 2012. pp. 353-361.

[16] Rusin Iu.S. Transformatori zvukovoi i ulitrazvukovoi chastoti. [Transformers at sound and ultrasound frequency]. «Energia», L., 1973. (In Russian).

[17] Hnikov A. V. Teoria i raschet mnogoobmotochnikh transformatorov [Theory and design of multiwinding transformers]. "SolomonPress", 2003. (In Russian).

[18] Quing Chen, F. C. Lee, Jian Zhong Jang, M. M. Jovanovic. A New Model for Multiple-Winding Transformer. IEE Power Electronics Specialists Rec., 1994, pp. 864-871.

[19] Tadeusz J. Sobczyk, Joseph EL Hayek. On parameters determination of multi-port equivalent scheme for multi-winding traction transformers. Archives of Electrical Engineering, vol. 64(1), pp. 1727 (2015). DOI 10.1515/aee-2015-0003. Cracow University of Technology. Institute on 
Electromechanical Energy Conversion, Warszawska 24, 31-155 Krakow, Poland.

[20] Joseph El Hayek, Tadeusz Sobczyk. Estimation of Parameters of Multi-Port Equivalent Scheme for Multi-Winding Traction Transformers. University of Applied Sciences and Arts Western Switzerland, School of Engineering, Sion. Zeszyty Problemowe Maszyny Elektryczne Nr 3/2014 (103).

[21] Wade G. Enright. Transformer models for electromagnetic transient studies with particular reference to HV DC transmission. Thesis presented for the degree of Doctor of Philosophy. University of Canterbury, Christchurch, New Zealand. February 1996.

[22] Carl Michael F. Odulio, Miguel T. Escoto, Jr. Multiple Winding Transformer Model for Power
Supply Applications in Circuit Simulations. Proceedings of the World Congress on Engineering 2013 Vol II, WCE 2013, July 3 - 5, 2013, London, U.K. ISBN: 978-988-19252-8-2, ISSN: 2078-0958 (Print).

[23] Ajay Gangupomu. Multi-Winding Model with Direct Extraction of Parameters from Voltage Measurements. A Thesis Presented to the Graduate School of the University of Florida. In Partial Fulfillment of the Requirements for The Degree of Master of Science. University of Florida, 2003.

[24] Beklemishev D.V. Dopolnitelinie glavi lineinoi algebri [Additional chapters of linear algebra]. “Nauka”, 1983. (In Russian).

\section{Сведения об авторах.}
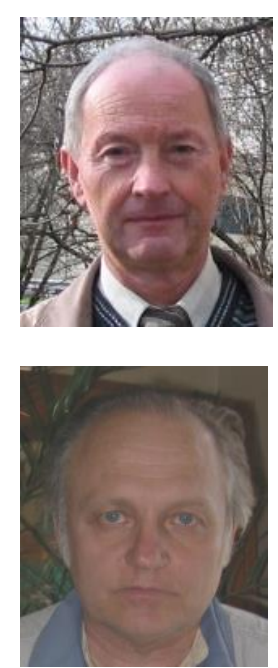

Бошняга В.А., окончил Кишиневский Политехнический институт в 1971 г. защитил диссертацию на степень кандидата технических наук в институте Электродинамики Академии наук Украины в 1988 г. ведущий научный сотрудник, область научных интересов связана с расчетами режимов электрических систем с использованием разрабатываемых моделей трансформаторных устройств.

e-mail: valeriu.bosneaga@gmail.com

Суслов В.М., окончил Кишиневский политехнический институт в 1972 г. Научный сотрудник, область научных интересов связана с электропередачами переменного тока повышенной пропускной способности, их влиянием на окружающую среду, режимами энергетических систем, переходными электромеханическими процессами, моделированием в энергетических системах.

E-mail: $\underline{\text { svictorm46@gmail.com }}$ 